\section{Rationale for \\ a pediatric-inspired approach in the adolescent and young adult population with acute lymphoblastic leukemia, with a focus on asparaginase treatment}

\author{
Carmelo Rizzari, ${ }^{1}$ Maria Caterina Putti, ${ }^{2}$ \\ Antonella Colombini, ${ }^{1}$ Sara Casagranda, ${ }^{1}$ \\ Giulia Maria Ferrari, ${ }^{1}$ \\ Cristina Papayannidis, ${ }^{3}$ Ilaria lacobucci, ${ }^{3}$ \\ Maria Chiara Abbenante, ${ }^{3}$ Chiara Sartor, \\ Giovanni Martinelli ${ }^{3}$ \\ 1Pediatric Hematology-Oncology Unit, \\ Department of Pediatrics, University of \\ Milan-Bicocca, Monza; ${ }^{2}$ Pediatric Onco- \\ Hematology Unit, Clinic of Pediatrics, \\ Padua; ${ }^{3}$ Institute of Hematology, \\ University of Bologna, Italy
}

\section{Abstract}

In the last two decades great improvements have been made in the treatment of childhood acute lymphoblastic leukemia, with 5-year overall survival rates currently approaching almost $90 \%$. In comparison, results reported in adolescents and young adults (AYAs) are relatively poor. In adults, results have improved, but are still lagging behind those obtained in children. Possible reasons for this different pattern of results include an increased incidence of unfavorable and a decreased incidence of favorable cytogenetic abnormalities in AYAs compared with children. Furthermore, in AYAs less intensive treatments (especially lower cumulative doses of drugs such as asparaginase, corticosteroids and methotrexate) and longer gaps between courses of chemotherapy are planned compared to those in children. However, although favorable results obtained in AYAs receiving pediatric protocols have been consistently reported in several international collaborative trials, physicians must also be aware of the specific toxicity pattern associated with increased success in AYAs, since an excess of toxicity may compromise overall treatment schedule intensity. Cooperative efforts between pediatric and adult hematologists in designing specific protocols for AYAs are warranted.

\section{Introduction}

Acute lymphoblastic leukemia (ALL) is the most common cancer of childhood. ${ }^{1,2}$
Improvements in overall survival (OS) in children with ALL are among the major successes in the history of cancer treatment. ${ }^{1,2}$ OS rates obtained in countries adopting modern intensive chemotherapy schedules are in fact in the range of $85-90 \% .{ }^{1,2}$ ALL represents almost $30 \%$ of all childhood cancers, but only $6 \%$ of cancers in adolescents and young adults (AYAs aged between 20 and 34 years). ${ }^{1}$ Survival rates in AYA patients are lower than in children, for example, 5 -year OS rates were $89 \%$ for children aged under 15 years versus $50 \%$ OS for those aged $15-19$ years in the early $2000 \mathrm{~s}^{1}{ }^{1}$ It has therefore been suggested that treatment of AYA patients should be closer to the strategies included in pediatric ALL trials, i.e. intensified post-remission strategies including high-dose chemotherapy agents (i.e. steroids, methotrexate) and intensive use of asparaginase (ASP).$^{1-8}$ In the main, adolescents tend to start receiving adult protocols at around age 18 years. ${ }^{4}$ Asparaginase are valuable agents widely used in the treatment of childhood ALL. Three forms are currently available: two are derived from $E$. coli (one native and its pegylated form, PEG-ASP) and one from Erwinia chrysanthemi (asparaginase Erwinia chrysanthemi; crisantaspase). ${ }^{8}$ These ASP products are not interchangeable due to their different pharmacological and antigenic properties; in addition, their use is associated with considerable variations in efficacy and toxicity depending on several factors such as the individual patient, the dosage/schedule adopted and also the ongoing line of treatment. ${ }^{8}$ The biological mechanism underlying ASP-related therapeutic effects is the same for all three forms, i.e. a deep and prolonged asparagine (ASN) depletion induced in plasma immediately after its administration induces apoptosis in leukemic blasts. ${ }^{9}$ Response to ASP varies from patient to patient; it has been suggested that the microenvironment of bone marrow-derived mesenchymal cells where leukemic cells grow has high levels of ASN-synthetase, up to 20times higher than the leukemic blast, and that ASN produced within the microenvironment may provide protection against ASP. ${ }^{10}$ Downregulation of ASN-synthetase could reduce the capacity of the microenvironment to protect against ASP, whilst upregulation of ASN- synthetase could conversely confer enhanced protection against ASP.

Allergic reactions or silent inactivation may develop, both of which may potentially reduce the therapeutic benefit of ASP. ${ }^{8}$ For this specific reason modern treatment protocols often include guidelines for timely identification of allergic reactions (and switch to another ASP product) and therapeutic drug monitoring (TDM) programs. The latter programs allow the early identification of patients with silent inactivation who do not benefit from current
Correspondence: Carmelo Rizzari, Pediatric Hematology-Oncology Unit, Department of Pediatrics, University of Milano-Bicocca, Via Pergolesi 33, 20900, Monza, Italy.

Tel. +39.039.233.3513 - Fax +39.039.230.1646.

E-mail: c.rizzari@hsgerardo.org

Key words: asparaginase, acute lymphoblastic leukemia, adolescent, young adult.

Acknowledgements: the authors would like to thank Tricia Dixon, JB Medical Ltd who provided medical writing support, funded by EUSA Pharma (an international division of Jazz Pharmaceuticals, plc).

Contributions: CR, MCP and GM wrote the preliminary text derived from presentations given in the frame of the European ALL Working Group (EWALL) meeting; TD wrote the first draft of the manuscript from the slides and a transcript of the presentations; the other authors reviewed the draft and made comments which were incorporated by TD to form a final draft.

Conflict of interests: CR is involved in scientific research supported by companies producing and/or marketing asparaginase products. The other authors declare no potential conflict of interests.

Funding: this work was also supported by: European LeukemiaNet, AIL, AIRC, Fondazione Del Monte di Bologna e Ravenna, Ateneo RF0 grants, PRIN 2010-2011, FP7- HEALTH-2012INNOVATION-1 NGS-PTL project, Comitato Maria Letizia Verga per lo Studio e la Cura della Leucemia Infantile, Monza, Italy.

Conference presentation: this paper summarizes the proceedings from a European ALL Working Group (EWALL) meeting held in Bologna (Italy) on March 15, 2013.

Received for publication: 15 July 2014 .

Revision received: 21 August 2014.

Accepted for publication: 25 August 2014.

This work is licensed under a Creative Commons Attribution NonCommercial 3.0 License (CC BYNC 3.0).

(C) Copyright C. Rizzari et al., 2014

Licensee PAGEPress, Italy

Hematology Reports 2014; 6:5554

doi:10.4081/hr.2014.5554

ASP treatment and facilitate a switch to a different ASP product. This switch ensures continued depletion of ASN, completion of the treatment schedule and maintenance of outcomes. ${ }^{8}$ This report summarizes the rationale for a pediatric-inspired approach in AYAs with ALL as presented and discussed during a symposium held in the framework of the 2013 European ALL Working Group (EWALL) International Meeting. A special effort to focus 
on how ASP treatment might contribute to achieve better results in AYAs was one of the aims of the symposium.

\section{Current guidelines in acute lymphoblastic leukemia: focus on adolescents and young adults}

Outcomes in patients with ALL vary by age and phenotype. ${ }^{2}$ Patients with B-cell ALL have better outcomes than those with T-cell ALL. Indeed, optimal outcomes are seen in children aged 1-5 years with B-cell ALL, with 10-year event free survival (EFS) of around 80\%. EFS falls to around 70\% in children with B-cell ALL aged 10 and over, in contrast EFS rates are somewhat less favorable in children with T-cell ALL but remain fairly static when older ages are concerned. ${ }^{2}$

Survival rates in AYAs are poor compared with those in younger children. Data from Surveillance Epidemiology and End Results (SEER) 2000-2004 reported 10-year OS of around $80 \%$ in children aged under 15 years, falling to $60 \%$ in adolescents aged $15-20$ years and $30 \%$ in young adults aged $20-30$ years; rates have improved by a further $10-15 \%$ over the past decade in the AYA group. The steepest decline in survival is seen in mid-adolescence, the sudden decrement at 18 years coincides with newly diagnosed patients receiving adult rather than pediatric regimens. ${ }^{4}$

Acute lymphoblastic leukemia can be challenging to treat in AYA. There is an increased incidence of unfavorable and decreased incidence of favorable cytogenetic abnormalities in adolescents compared with children (Table 1). ${ }^{1}$ As we will discuss later in this paper, data from adult cooperative groups demonstrates improved outcomes in AYAs treated with intensified post-remission strategies as per pediatric regimens. ${ }^{5}$ However, there is a lack of European guidance for the treatment of AYA patients, although the US-based National Comprehensive Cancer Network (NCCN) Clinical Practice Guidelines in Oncology (NCCN Guidelines) do provide guidance and consider AYA separately from the adult population. ${ }^{6}$

\section{National Comprehensive Cancer Network guidelines}

National Comprehensive Cancer Network is an alliance of 25 US-based cancer centers, which work together to develop treatment guidelines and carry out research into cancer. The NCCN Guidelines provide recommendations based on the best evidence available at the time they are derived. The guidelines are continuously updated and revised to reflect new data and clinical information; however, they are not necessarily directly reflective of established practice. The guidelines define AYAs as aged 15-39 years and are further subdivided by the presence of the Philadelphia $\mathrm{Ph}$ ) chromosome into $\mathrm{Ph}$-positive ALL and $\mathrm{Ph}$ negative ALL. ${ }^{6}$

The NCCN guidelines recommend pediatricinspired chemotherapy regimens for Ph-positive and Ph-negative ALL in AYAs for induction as outlined below. Maintenance therapy consisting of weekly methotrexate plus daily 6 mercaptopurine (6-MP) plus monthly vincristine/prednisone pulses (for 2-3 years) is recommended, with the addition of tyrosine kinase inhibitors (TKI) (imatinib or dasatinib) in Ph-positive patients. ${ }^{6}$

\section{Ph-positive disease}

Patients should be treated in a clinical trial whenever possible. In the absence of an appropriate clinical trial, induction therapy should be a pediatric-inspired multi-agent chemotherapy combined with a TKI. Treatment regimens should include adequate central nervous system prophylaxis for all patients. In those patients achieving a complete response (CR) following initial induction therapy, consolidation with allogeneic hematopoietic stem cell transplantation (HSCT) should be considered if a matched donor is available. ${ }^{6}$ Emerging data suggests that in younger AYA patients (aged $\leq 21$ years), allogeneic HSCT may not confer an advantage over chemotherapy plus TKIs, ${ }^{11}$ and long-term data is eagerly awaited to determine whether younger patients can be successfully treated without allogeneic HSCT. After HSCT, TKI should be considered. For patients without a donor, consolidation therapy following a CR is a continuation of multi-agent chemotherapy plus a TKI. Such patients should continue to receive post-consolidation maintenance therapy with a regimen including a TKI. ${ }^{6}$

Adolescents and young adults patients with Ph-positive relapsed/refractory ALL should participate in a clinical trial. In the absence of an appropriate trial, the patient may be consid- ered for second-line therapy with multi-agent chemotherapy combined with an alternative TKI, allogeneic HSCT (if a second CR is achieved) or donor lymphocyte infusion, if the patient relapses after allogeneic HSCT. ${ }^{6}$

\section{Ph-negative disease}

Patients should be treated in a clinical trial whenever possible. In the absence of an appropriate trial, induction therapy should be based on pediatric-inspired protocols. Treatment regimens should include adequate central nervous system prophylaxis for all patients. Testing for TPMT gene polymorphism should be considered for patients receiving 6-MP as part of maintenance therapy, especially in patients who experience severe bone marrow toxicities. Monitoring for minimal residual disease (MRD) should be considered in patients achieving CR after initial induction therapy. ${ }^{6}$

In patients achieving $\mathrm{CR}$, multi-agent based chemotherapy in consolidation, re-induction and maintenance phases must be given. If a matched donor is available, consolidation with allogeneic HSCT may be considered, particularly for patients with residual disease as assessed by MRD, or with high-risk features. In patients achieving less than CR after initial induction therapy, the treatment approach is similar to patients with relapsed/refractory ALL. ${ }^{6}$ For patients with relapsed/refractory disease following an initial CR, the approach to second-line treatment depends on the duration of the initial response. In patients with a late relapse (i.e., relapse occurring $\geq 36$ months from initial diagnosis) re-treatment with the same induction regimen may be reasonable. Participation in a clinical trial is preferred; in the absence of an appropriate trial consider second-line therapy with previously unused induction regimens, salvage chemotherapy, allogeneic HSCT (if a second CR is achieved). ${ }^{6}$

\section{GIMEMA ALL 1308}

The Gruppo Italiano Malattie EMatologiche

Table 1. Cytogenetic and immunophenotypic features of acute lymphoblastic leukemia in adolescents and young adults and in children.

\begin{tabular}{lccc} 
Feature & Prognostic value & Pediatric ALL & AYA ALL \\
Ph chromosome & Negative & Rare & Uncommon $(3 \%)$ \\
t(12;21) & Positive & Common $(25 \%)$ & Rare \\
\hline Hyperdiploidy & Positive & Common $(20 \%)$ & Less common \\
B-cell ALL & Positive & More common & Less common \\
\hline T-cell ALL & Negative & $10-15 \%$ & $25 \%$ \\
\hline
\end{tabular}

ALL, acute lymphoblastic leukemia; AYA, adolescents and young adults. 
dell'Adulto (GIMEMA) protocol provides an example of current treatment in AYAs in Europe. Patients are included if they are aged between 18 and 35 years, with a diagnosis of non-B-mature, Ph-negative ALL. ${ }^{7}$

All patients receive steroids and methotrexate prior to induction therapy. Patients receive induction Ia (vincristine, daunorubicin, ASP and prednisone) followed by induction Ib (cyclophosphamide, 6-MP and cytarabine). Patients who achieve hematological remission proceed to consolidation therapy. Patients receive consolidation therapy according to their risk group. Standard-risk patients receive high-dose methotrexate and 6-MP. High-risk patients receive consolidation therapy in three steps; step 1 (dexamethasone, vincristine, methotrexate, cytarabine and ASP), step 2 (dexamethasone, vindesine, methotrexate, ifosfamide, ASP and daunorubicin hydrochloride) and step 3 (dexamethasone, cytarabine and ASP). Two step re-induction follows consolidation: IIa (vincristine, doxorubicin hydrochloride, ASP and dexamethasone) and IIb (cyclophosphamide, thioguanine and cytarabine) ${ }^{7}$ Early data from GIMEMA ALL 1308 presented at the European Hematology Association meeting in 2014 suggest that the intensified protocol is effective and well tolerated in AYA. ${ }^{12}$ Sixty-six patients have been enrolled into GIMEMA ALL 1308, of whom 61 were eligible for treatment. Complete response rate was $98 \%$, which is higher than that found in previous studies GIMEMA ALL 2000 (84\%) and GIMEMA ALL 0904 (83\%). At 24 months OS in GIMEMA ALL 1308 was $72.3 \%$ compared with $61 \%$ and $72 \%$ in the earlier GIMEMA studies. Severe adverse events have been reported in 11 patients (18\%), ASP-related adverse events accounted for six serious adverse events and infection for the other five.

\section{Asparaginase in children, ado- lescents and young adults}

The ASPs are a universal component of ALL therapy and are used for remission induction and intensification treatment in every pediatric regimen for ALL. ${ }^{8}$

Leukemic cells are unable to synthesize asparagine (ASN) and rely on extracellular sources. In the presence of ASP, ASN is rapidly de-aminated in serum depleting extracellular sources and reducing the supply of ASN to leukemic cells. Leukemic cells are unable to undertake protein biosynthesis leading to cell death. ${ }^{9}$ Studies using intensive ASP have revealed significant benefit in terms of EFS, ${ }^{13,14}$ disease free survival (DFS) ${ }^{15}$ and continuous complete remission rate, ${ }^{16}$ when compared with less intensive ASP treatment. Also the completion of the treatment schedule is essential to ensure the expected full clinical benefit. In a study carried out by the DanaFarber Clinical Institute (DFCI), children were treated with an extended 30 weeks of highdose ASP during intensification $(\mathrm{n}=352)$. At 5 year follow-up, EFS in children who received less than 25 weeks of planned ASP therapy was significantly poorer than in those who received 26 weeks or more of therapy: $73 \%$ versus $90 \%$, $\mathrm{P}<0.01 .^{13}$ A significant improvement in EFS with continued ASP therapy was also seen in a retrospective analysis by the Tokyo Children's Cancer Study Group, wherein children who received more than $50 \%$ of the scheduled dose had a significantly improved 5 -year EFS versus those who received less than $50 \%$ of the scheduled dose: $92.9 \%$ versus $74.1 \%, \mathrm{P}<0.025 .^{14}$

\section{Use of pediatric protocols in adolescents and young adults}

There is considerable evidence from retrospective analyses that treating AYAs with a pediatric protocol may improve clinical outcomes compared with treatment adopted in adult protocols..$^{3,5,17-20}$ Pediatric protocols have higher cumulative dosing of drugs (ASP, corticosteroids, methotrexate, vinca-alkaloids) and shorter gaps between courses of chemotherapy compared with adult protocols. ${ }^{17}$

A systematic review and meta-analysis of comparative trials of AYA patients receiving induction therapy with either adult or pediatric-inspired chemotherapy identified 11 trials $(\mathrm{n}=2489)$. The AYA patients receiving a pediatric-inspired regimen had a significantly lower all cause mortality at 3 years compared to those receiving an adult regimen: relative risk $0.58,95 \% \mathrm{CI} 0.51-0.67, \mathrm{P}<0.05 .{ }^{21}$ The absolute risk reduction for all cause mortality at 3 years was 0.2 and the number needed to treat to prevent one death with pediatricinspired regimens was 5 (95\%CI $4-7)$. Secondary end-points included all cause mortality at the end of the trial, complete remission, 3-year EFS and relapse rate. Significant benefit was seen in the patients receiving the pediatric-inspired regimen $(\mathrm{P}<0.05$ for all secondary end-points). Non-relapse mortality was similar in both groups.

The German multicenter ALL (GMALL) protocols were originally based on pediatric Berlin-Frankfurt-Munster (BFM) protocols and have been optimized for AYAs since 1981. A retrospective analysis compared outcomes from GMALL 05/93 (an earlier study) and GMALL 07/03 (a later study). The main innovations in GMALL 07/03 were intensified shortened induction with dexamethasone rather than with prednisone, PEG-ASP rather than native ASP, intensified first consolidation, six doses of high dose methotrexate and ASP during consolidation, matched unrelated SCT for high risk and very high risk patients without sibling donor and SCT indication in patients with persistent minimal residual disease. AYA patients receiving the later protocol (GMALL 07/03) had significant improvements in 5-year OS compared with GMALL 05/93 (65\% in GMALL 07/03 versus $46 \%$ in GMALL 05/93). This data represents the largest cohort of AYA patients treated to date with pediatric-inspired protocols (642 in GMALL 05/93 and 887 in GMALL 07/03). ${ }^{22}$

A number of other studies have been carried out using retrospective data to compare outcomes in AYAs receiving pediatric and adult inspired protocols. The results are shown in Table 2 and demonstrate that outcomes are significantly improved in AYA patients receiving a pediatric-inspired protocol compared with an adult-inspired protocol.

A retrospective study compared outcomes in 177 AYAs aged 15-20 years entering either a pediatric [French Acute Lymphoblastic Leukemia Group (FRALLE)-93] or an adult protocol [Leucémie Aiguë Lymphoblastique de l'Adulte (LALA)-94]. The cumulative doses of treatment (vincristine/vindesine, prednisone, dexamethasone, ASP, daunorubicin/ doxorubicin/mitoxantrone, vepeside/cyclophosphamide) were higher in the pediatric protocol than in the adult protocol. The overall dose of ASP was 20-times higher in the pediatric protocol: $180,000 \mathrm{IU} / \mathrm{m}^{2}$ in the pediatric regimen versus $9000 \mathrm{IU} / \mathrm{m}^{2}$ in the adult regimen. ${ }^{18}$

A retrospective study compared outcomes in adolescents aged 14-18 years treated on the pediatric Associazione Italiana Ematologia Oncologia Pediatrica (AIEOP) ALL 95 and 2000 protocols with those treated on adult GIMEMA ALL 0496 and 2000 protocols. ${ }^{19}$ The pediatric protocols had seven-drug induction followed by risk-modulated post-remission therapy. SCT was recommended for very high-risk patients. Another retrospective study compared the Dutch Children's Oncology Group (DCOG) pediatric regimen with the Hemato-Oncologie voor Volwassenen Nederland (HOVON) adult protocols in AYAs. The main differences between the regimens were shorter intervals between courses ( $\leq 1$ week versus $\leq 4$ weeks) and more ASP (mean cumulative dosage: $101,000 \mathrm{IU} / \mathrm{m}^{2}$ versus $70,000 \mathrm{IU} / \mathrm{m}^{2}$ ) in the pediatric regimen. ${ }^{20}$

A similar study compared the Children's Cancer Group (CCG) pediatric regimen with the adult Cancer and Leukemia Group B (CALGB) regimen. The pediatric protocol included higher doses of ASP $\left(54,000 \mathrm{IU} / \mathrm{m}^{2}\right.$ versus $36,000 \mathrm{IU} / \mathrm{m}^{2}$ in induction and 90,000 or $318,000 \mathrm{IU} / \mathrm{m}^{2}$ versus $36,000 \mathrm{IU} / \mathrm{m}^{2}$ in postremission) and intrathecal methotrexate and 
cytarabine..$^{5}$ Although ASP is a pivotal drug in the treatment of ALL, protocols based on strategies not including ASP have also demonstrated benefit in AYAs. A recent study compared outcomes in 85 patients aged 12-40 years with PhALL treated with the pediatric augmented BFM regimen with outcomes in 71 historical controls who received hyper-CVAD (cyclophosphamide, vincristine, doxorubicin and dexamethasone). Outcomes [3-year complete remission duration (CRD) and OS] were comparable between the two groups: CRD was 70\% in the BFM arm versus $66 \%$ in the hyper-CVAD arm and $0 \mathrm{~S} 74 \%$ versus $71 \%{ }^{23}$

Toxicity is a key issue in the use of pediatric-inspired regimens in older patients, and may limit the potential benefit of high intensity pediatric-inspired regimens. Toxicity may has the potential to lead to increased adverse events, potentially lethal toxicities and a reduction in the total dose due to dose interruption, dose reduction or early cessation of therapy. Some recent protocols include monitoring for MRD to inform clinical decisions and ensure treatment intensity is appropriate to each individual's needs, whilst maintaining efficacy and minimizing adverse events.

Recent evidence presented at the American Society of Hematology (ASH) meeting in December 2013 suggests that pediatricinspired protocols are feasible and well tolerated in AYA patients. ${ }^{24,25}$ Data from the prospective US intergroup trial C10403 in 318 AYA patients aged 16-39 years receiving a pediatric-inspired regimen revealed grade 3-5 adverse events as follows: hyperglycemia (29.3\%), febrile neutropenia (19.2\%), hyperbilirubinemia (15.9\%), thrombosis $(2.9 \%)$, pancreatitis (1.1\%) and allergic reaction (0.7\%) in induction..$^{24}$ Data was also presented from UK Acute Lymphoblastic Leukemia (UKALL) 2003, a large study of 3129 patients, which included 229 patients aged 16-24 years, 56 of whom were aged 20 years or above. Most observed toxicities were more common in patients aged over 10 years than in younger patients, however, there was no difference in incidence between patients aged 16-24 years and 10-15 years. Age did not appear to impact on some adverse events (infection, hypersensitivity to ASP, neurotoxicity to vincristine), however, the incidence of thrombotic events due to ASP increased with increasing patient age and avascular necrosis was most commonly observed in patients aged 10-19 years..$^{25}$ Further data on toxicity comes from the Erwinaze Master Treatment Protocol (EMTP) was a compassionate use program which allowed patients with a grade 2 or higher hypersensitivity reaction to PEG-ASP or native ASP to switch to crisantaspase (trade named Erwinaze in the US). Adverse event reports or case report forms were completed for 940 patients aged 0-76 years (mean 9.7 years), of whom 16\% ( $\mathrm{n}=147)$ were aged $16-39$ years. Post hoc analyses revealed that the adverse event profile in the AYA population was consistent with the profile in the full population in this trial. ${ }^{26}$ However, despite the potential benefits of a pediatric regimen as shown in Table 2 , many AYA patients do not currently receive a pediatric regimen. This may be due to a number of factors including fear of increased incidence of side-effects and the potential for increased mortality due to toxicity, referral patterns (AYAs are referred by oncologists preferably to adult rather than pediatric centers), cost of treatment, lack of insurance (where applicable), lack of parental vigilance and poor compliance, lack of information, guidance and patient involvement in the decision about where to be treated. ${ }^{3,27,28}$

\section{Management of toxicities with asparaginase}

Asparginase treatment is associated with a number of adverse events, ${ }^{29}$ which can lead to discontinuation of ASP or delay in treatment and a reduction in clinical benefit.

A retrospective analysis reviewed the records of 214 patients aged 15-59 years with ALL or lymphoblastic lymphoma and considered the reasons for early discontinuation of ASP. ${ }^{30}$ All patients received six doses of native E. coli ASP during induction. ASP was delayed in $22 \%$ of patients and the number of doses was reduced in $41 \%$. The most common reasons for delay were coagulation abnormalities (47\%) and logistical reasons (34\%). The most common reasons for dose reduction were coagulation abnormalities (35\%), liver toxicity (17\%), logistical reasons (16\%) and pancreatic reaction (12\%). ${ }^{30}$

The majority of data on adverse events with ASP is from studies using E. coli-derived ASP. Different definitions of adverse events make it very difficult to compare data across studies; however, the pegylated formulation has reduced immunogenicity and consequently lower rates of hypersensitivity. ${ }^{31}$

Table 3 provides key information on adverse events and management strategies. ${ }^{4,29,31-39}$

Two adverse events, hypersensitivity and coagulation disorders, are discussed in greater depth below.

\section{Hypersensitivity}

Asparginase use may lead to the development of anti-ASP antibodies, which may result in a clinical hypersensitivity reaction or be symptom-free (known as silent inactivation). ${ }^{8}$

Hypersensitivity is the most commonly reported adverse reaction with all ASP; incidence varies according to a number of factors including type of ASP, dosing schedule, route of administration, concomitant medication and duration of treatment. ${ }^{8,29,40}$ Rates can be as high as $36 \%$ with native $E$. coli ASP and tend to be lower with PEG-ASP and crisantaspase. ${ }^{41}$ Silent inactivation occurs in around 30\% of patients receiving native $E$. coli $\mathrm{ASP}$ and rates are lower with PEG-ASP and crisantaspase.

There is cross-reactivity between $E$. coliderived ASP (native ASP and PEG-ASP) but not between $E$. coli-derived ASPs and crisantaspase, which is derived from Erwinia chrysanthemi. ${ }^{42}$ Therefore, it has been suggested that a change to crisantaspase in cases of allergy to native or pegylated $E$. coli ASP might ensure advantages in continuation of treatment and clinical benefit. ${ }^{40}$

Two recent studies demonstrate the adverse event profile seen with crisantaspase, given as second-line treatment to patients with a hyper-

Table 2. Outcomes in adolescents and young adults patients receiving a pediatric or an adult regimen. The comparison of complete response, event free survival and overall survival rates is reported as pediatric vs adult protocols, respectively.

\begin{tabular}{|c|c|c|c|c|c|c|}
\hline Country (years of recruitment) & Age range & Pediatric protocols & Adult protocols & CR (\%) & EFS (\%) & OS $(\%)$ \\
\hline France $^{18}(1993-2000)$ & $15-20$ & FRALLE-93 (n=77) & LALA-93 $(n=100)$ & $\begin{array}{l}94 \text { vs } 83 \\
\mathrm{P}=0.04\end{array}$ & $\begin{array}{l}67 \text { vs } 41 \\
\mathrm{P}<0.001\end{array}$ & $\begin{array}{l}78 \text { vs } 45 \\
\mathrm{P}<0.001\end{array}$ \\
\hline Italy $^{19}(1996-2003)$ & $14-18$ & AIEOP $95+2000(n=150)$ & GIMEMA 0496 + $2000(n=95)$ & 94 us 89 & & 80 us 71 \\
\hline Netherlands ${ }^{20}(1984-2004)$ & $15-18$ & DCOG ALL6+9 (n=47) & HOVON ALL-5 + ALL-18 $(\mathrm{n}=44)$ & $\begin{array}{l}98 \text { vs } 91 \\
\mathrm{P}=0.19\end{array}$ & $\begin{array}{l}69 \text { vs } 34 \\
\mathrm{P}<0.001\end{array}$ & $\begin{array}{l}79 \text { vs } 38 \\
\mathrm{P}<0.001\end{array}$ \\
\hline $\mathrm{USA}^{5}(1988-2001)$ & $16-20$ & CCG $1882+1901(\mathrm{n}=197)$ & $\begin{array}{c}\text { CALGB 8811+9111+ } 9311 \\
+9511+19802(\mathrm{n}=124)\end{array}$ & $\begin{array}{l}90 \text { vs } 90 \\
\mathrm{P}=0.89\end{array}$ & $\begin{array}{l}63 \text { vs } 34 \\
\mathrm{P}<0.001\end{array}$ & $\begin{array}{l}67 \text { us } 46 \\
\mathrm{P}<0.001\end{array}$ \\
\hline
\end{tabular}

CR, complete response; EFS, event free survival; OS, overall survival. 
sensitivity reaction to $E$. coli-derived ASP. In EMTP, the most frequently reported adverse events included hypersensitivity (13.6\%), local hypersensitivity reaction (3.3\%) and anaphylaxis $(0.9 \%)$, infection/sepsis $(3.9 \%)$, pancreatitis (3.9\%), fever (3.8\%), hyperglycemia (3.7\%) and increased transaminases $(3.5 \%) .^{26}$ In AALL07P2, which enrolled 55 patients, hypersensitivity was seen in $10.9 \%$ of patients, hyperglycemia in $10.9 \%$ and pancreatitis in $1.8 \%{ }^{43}$ The safety profile of second-line crisantaspase seems to compare favorably with that observed in patients treated with first-line native $E$. coli ASP; however, the limitations of these two studies (small numbers in one and the compassionate-use design in the other) should be taken into consideration and confirmed in larger cohorts and prospective studies.

\section{Coagulation disorders}

Reduced protein synthesis with ASP leads to falls in the serum levels of key proteins. Reduced serum albumin levels impact on the clearance and metabolism of some agents, including steroids, with a potential reduction in efficacy. ${ }^{44,45}$ Reduced serum levels of immunoglobulins and lectins may also increase the risk of infection.

Coagulation disorders result from the effect of ASP on protein synthesis, which leads to reductions in plasminogen, fibrinogen, antithrombin, protein $\mathrm{C}$ and $\mathrm{S}$, factors IX and $\mathrm{X}$. Reductions in anti-coagulant proteins can impair thrombin inhibition or result in elevated thrombin levels which may increase the risk of bleeding or thrombosis. Therefore, ASP treatment has been associated with an increased risk of thrombo-hemorrhagic disorders. Thrombosis, mostly at venous sites, is considered the main risk. ${ }^{29}$
Coagulation disorders may occur in up to one-third of patients receiving ASP and generally occur early in treatment. ${ }^{29}$ It is difficult to compare rates across the ASPs, however, data from EMTP and AALL07P2 showed low rates of thrombosis/hemorrhage with crisantaspase ( $0 \%$ in AALL07P2, ${ }^{43}$ thrombosis rates of $2.1 \%$ and hemorrhage rates of $1 \%$ in EMTP) ${ }^{26}$ In adults, work has shown that a single dose of PEG-ASP leads to a reduction in plasma antithrombin III to $<50 \%$ of normal in twothirds of patients (16/25), with an overall median nadir level of $45 \%$ of normal. The reduction in plasma antithrombin III lasted for approximately 21 days, suggesting that there may be greater potential for thrombotic adverse events with PEG-ASP due to an extended duration of asparagine depletion. ${ }^{46}$ As with all thrombotic conditions, rates are higher in adults than in children., ${ }^{47}$ A review of 548 ALL patients treated at the DFCI between 1991 and 2008 revealed that venous thrombotic events (VTE) occurred in $8 \%$ of patients, including $5 \%$ of pediatric patients and $34 \%$ of adult patients (18-50 years). ${ }^{47}$ Median time to VTE in this study was 3.5 months (0.5-10.1 months) with no difference between adult and pediatric patients.

Risk factors for VTE include older age at diagnosis, T-cell phenotype, high-risk ALL, use of a central line, steroid treatment, presence of mediastinal mass and inherited thrombophilia traits. ${ }^{29,47-49}$ Older children and adolescents (15-20 years) have a increased risk of thrombosis compared with younger children (6-10 years), odds ratio: 4.0 versus $11,7, \mathrm{P}<0.01 .^{47}$ However, although changes in coagulation proteins are commonly observed during ASP treatment, one recent study failed to show a clear association between coagulation derangement and the occurrence of VTE in a pediatric population. ${ }^{50}$ VTE are severe events with significant morbidity and mortality. Inpatients receiving ASP at DFCI between 1998 and 2008 the most common complication was VTE recurrence, which occurred in $44 \%$ of adults and $15 \%$ of children. ${ }^{47}$

Thrombosis has an adverse impact on outcome. In a retrospective analysis of 214 patients aged 15-59 years with ALL or lymphoblastic leukemia, treated with six doses of native $E$. coli ASP during induction, 9.8\% of patients experienced a thrombotic event during induction. ${ }^{30}$ Treatment with ASP was stopped in 10 of the patients who experienced a thrombosis. Patients who experienced thrombosis had significantly poorer outcomes compared with those without thrombosis; 7year OS of 19 months versus 53 months, $\mathrm{P}=0.06$. Therefore, strategies to prevent VTE and allow administration of adequate doses of ASP are warranted but there is still no consensus on which diagnostic and prophylactic strategies should be used to prevent them. Prophylactic administration of antithrombin concentrates and the use of heparin have been attempted but results and indications are still far from consistent. ${ }^{48,51}$

Recommendations suggest that in general, ASP should be withheld i) in case of severe VTE (Grade 3-4) and discontinued if symptoms do not resolve or ii) in cerebral thrombosis. In resolved non-cerebral VTE, resumption of ASP at lower doses may be tried under anticoagulation treatment. ${ }^{4}$

Re-exposure to ASP has been indeed safely performed. ${ }^{47,51}$ A retrospective analysis of 1824 patients enrolled in UKALL 2003 aged 1-25

Table 3. Main toxicities related to asparaginase treatment.

\begin{tabular}{|c|c|c|c|}
\hline & Incidence & pact & lanagement options \\
\hline Hyperglycemia & $\begin{array}{l}\text { 20-35\% children } \\
\text { 25\% } \\
\text { 25dults } \\
34\end{array}$ & $\begin{array}{l}\text { Increased infection rates } \\
\text { and poor survival outcomes } \\
33,35\end{array}$ & $\begin{array}{l}\text { Resolves within 2-4 weeks, }{ }^{31} \text { treat with insulin therapy, }{ }^{29} \text { glycemic control should be } \\
\text { improved using diet and exercise, }{ }^{29} \text { monitoring of blood glucose essential to ensure } \\
\text { that patients are identified }{ }^{29}\end{array}$ \\
\hline Pancreatitis & $\begin{array}{l}5-10 \% \text { of patients, } 31,36 \\
\text { rates similar in adults } \\
\text { and children }\end{array}$ & $\begin{array}{l}\text { Generally mild, but can present as a severe } \\
\text { complication, } 37 \text { long-term sequelae include } \\
\text { the formation of pancreatic cysts and } \\
\text { persistent diabetes mellitus }{ }^{37}\end{array}$ & $\begin{array}{l}\text { Bowel rest (tube feeding), correction of electrolytes and glucose disturbances and } \\
\text { prophylactic antibiotic treatment. Use of octreotide and protease inhibitors has also been } \\
\text { suggested; }{ }^{29} \text { ASP treatment must be discontinued in patients with symptomatic } \\
\text { pancreatitis }{ }^{29}\end{array}$ \\
\hline Liver toxicity & $\begin{array}{l}\text { 20\% children }{ }^{4} \\
33 \% \text { adults }^{4,29}\end{array}$ & $\begin{array}{l}\text { Commonly presents as elevation of liver enzymes } \\
\text { (aspartate transaminase and alanine transaminase), } \\
\text { but may also present as hyperbilirubinemia }{ }^{4} \\
\text { and reduction of hepatic protein synthesis, }{ }^{29}\end{array}$ & $\begin{array}{l}\text { Test liver function prior to each ASP dose and when drugs metabolized by the liver e.g. } \\
\text { anthracycline and vinca alkaloids, are given after ASP; } 4 \text { ASP treatment should be } \\
\text { interrupted in patients with Grade } 3 \text { or } 4 \text { liver toxicity and resumed with careful } \\
\text { monitoring if toxicity resolves to Grade } 1^{4}\end{array}$ \\
\hline
\end{tabular}

\begin{tabular}{ll}
$\begin{array}{l}\text { Serum amylase } \\
\text { and lipase } \\
\text { changes }\end{array}$ & - \\
& \\
\hline $\begin{array}{l}\text { Elevation of } \\
\text { plasma } \\
\text { triglycerides }\end{array}$ & May mask initial signs of pancreatic distress; \\
\hline
\end{tabular}

Monitor during treatment; 4 withhold treatment if levels increase to $>2-3$ times the upper limit of normal, ${ }^{4}$ discontinue if levels continue to be $>3$ times the upper normal limit for more than 2-3 days; ${ }^{4}$.rechallenge may be possible, but only for very mild cases (e.g. asymptomatic cases only and resolving within 48 hours)

plasma

tiogcerides
Treatment is poorly defined and may include a wide range of measures, i.e. from none to concomitant treatment and dietary modifications, hydration, use of lipid-lowering agents or even plasmapheresis ${ }^{38,39}$ 
years revealed a thrombotic event rate of $3.2 \%{ }^{52}$ Fifty of the 59 patients with thrombotic events required ongoing ASP and 38 (73\%) were re-exposed to PEG-ASP, including 10 patients with cerebral venous sinus thrombosis. There was no recurrence of thrombosis during re-exposure and no excess bleeding due to heparin. Low molecular weight heparin was used during re-exposure in three-quarters of patients. In the DFCI study, ${ }^{47}$ ASP was withheld after diagnosis of VTE for a median of 9 weeks in children and 4 weeks in adults. ASP was restarted in $77 \%$ of patients and most (70\%) received at least $85 \%$ of the scheduled dose of ASP. Recurrence of VTE occurred in $33 \%$ of patients restarted on ASP. There was no significant difference in clinical outcomes in the patients with VTE compared to those without VTE; 2 -year OS of $86 \pm 7 \%$ versus $95 \pm 1 \%$, $\mathrm{P}=0.12$. There were no deaths directly related to VTE in either group.

Hematologists should be aware of possible treatment complications with ASP; careful vigilance can lead to necessary modulation and safe completion of treatment.

\section{Conclusions}

In conclusion, the design of modern chemotherapy protocols for AYA should be the result of cooperative efforts between pediatric and adult hematologists. It is important to consider the specific biological and response patterns of ALL subtypes affecting AYA and also their well known propensity to develop severe side-effects. In this context, ASP may represent a great opportunity, given its specific mechanism of action, the possibility of effective TDM and the established pattern of toxicity. Toxicity with ASP is easily preventable with careful ASP treatment dosage modulation and manageable with advanced supportive treatment currently available to hematologists.

\section{References}

1. Mohan SR, Advani AS. Treatment of acute lymphoblastic leukemia in adolescents and young adults. JAYAO 2011;1:19-24.

2. Pui CH, Schrappe M, Ribeiro RC, et al. Childhood and adolescent lymphoid and myeloid leukemia. Hematology Am Soc Hematol Educ Program 2004:118-45.

3. Schafer ES, Hunger SP. Optimal therapy for acute lymphoblastic leukemia in adolescents and young adults. Nat Rev Clin Oncol 2011;8:417-24.

4. Stock W, Douer D, Deangelo DJ, et al. Prevention and management of asparaginase/pegasparaginase-associated toxici- ties in adults and older adolescents: recommendations of an expert panel. Leuk Lymphoma 2011;52:2237-53.

5. Stock W, La M, Sanford B, et al. What determines the outcomes for adolescents and young adults with acute lymphoblastic leukemia treated on cooperative group protocols? A comparison of Children's Cancer Group and Cancer and Leukemia Group B studies. Blood 2008;112:1646-54.

6. National Comprehensive Cancer Network Clinical Practice Guidelines in Oncology. Acute lymphoblastic leukemia. 2012. Available from: http://www.nccn.org/professionals/physician_gls/f_guidelines.asp

7. U.S. National Institutes of Health. Combination chemotherapy in treating young adult patients with acute lymphoblastic leukemia (LAL1308). Available from: http://clinicaltrials.gov/show/ NCT01156883.

8. Pieters R, Hunger S, Boos J, et al. Lasparaginase treatment in acute lymphoblastic leukemia. Cancer 2011;117:23849.

9. Müller HJ, Boos J. Use of L-asparaginase in childhood ALL. Crit Rev Oncol Hematol 1998;28:97-113.

10. Iwamoto S, Mihara K, Downing JR, et al. Mesenchymal cells regulate the response of acute lymphoblastic leukemia cells to asparaginase. J Clin Invest 2007;117:104957.

11. Schultz KR, Bowman WP, Aledo A, et al. Improved early event-free survival with imatinib in Philadelphia chromosome-positive acute lymphoblastic leukemia: a children's oncology group study. J Clin Oncol 2009;27:5175-81.

12. Testi AM, Piciocchi A, D’Angiò et al. Italian GIMEMA 1308 protocol treatment of acute lymphoblastic leukemia (ALL) in adolescents and young adults (AYA): intensification of treatment based on the pediatric AIEOP ALL 2000 protocol. Abstract presented at the 19th Congress of the European Hematology Association, Milan, 2014.

13. Silverman LB, Gelber RD, Dalton VK, et al. Improved outcome for children with acute lymphoblastic leukemia: results of DanaFarber Consortium Protocol 91-01. Blood 2001;97:1211-8.

14. Ogawa C, Ohara A, Manabe A, et al. Tokyo Children's Cancer Study Group (TCCSG) study L99-15. Blood 2005;106:Abstract 878.

15. Pession A, Valsecchi MG, Masera G, et al. Long-term results of a randomized trial on extended use of high dose L-asparaginase for standard risk childhood acute lymphoblastic leukemia. J Clin Oncol 2005;23:7161-7.

16. Amylon MD, Shuster J, Pullen J, et al. Intensive high-dose asparaginase consolidation improves survival for pediatric patients with $\mathrm{T}$ cell acute lymphoblastic leukemia and advanced stage lymphoblastic lymphoma: a Pediatric Oncology Group study. Leukemia 1999;13:335-42.

17. Rijneveld AW, van der Holt B, Daenen SM, et al. Dutch-Belgian HOVON Cooperative group. Intensified chemotherapy inspired by a pediatric regimen combined with allogeneic transplantation in adult patients with acute lymphoblastic leukemia up to the age of 40. Leukemia 2011;25:1697-703.

18. Boissel N, Auclerc MF, Lhéritier V, et al. Should adolescents with acute lymphoblastic leukemia be treated as old children or young adults? Comparison of the French FRALLE-93 and LALA-94 trials. J Clin Oncol 2003;21:774-80.

19. Testi AM, Valsecchi MG, Conter V, et al. Difference in outcome of adolescents with acute lymphoblastic leukemia (ALL) enrolled in pediatric (AIEOP) and adult (GIMEMA) protocols. Blood 2004;104: Abstract1954.

20. de Bont JM, Holt B, Dekker AW, et al. Significant difference in outcome for adolescents with acute lymphoblastic leukemia treated on pediatric vs adult protocols in the Netherlands. Leukemia 2004;18:2032-53.

21. Ram R, Wolach 0 , Vidal $\mathrm{L}$, et al. Adolescents and young adults with acute lymphoblastic leukemia have a better outcome when treated with pediatric-inspired regimens: systematic review and metaanalysis. Am J Hematol 2012;87:472-8.

22. Gokbuget $N$, Beck J, Brandt $K$, et al. Significant improvement of outcome in adolescents and young adults (AYAs) aged 15-35 years with acute lymphoblastic leukemia (ALL) with a pediatric derived adult ALL protocol: results of 1529 AYAs in 2 consecutive trials of the German Multicenter Study Group for Adult ALL (GMALL). Presented at 55th ASH Annual Meeting, New Orleans, December 2013.

23. Rytting ME, Thomas DA, O'Brien SM, et al. Augmented Berlin-Frankfurt-Münster therapy in adolescents and young adults (AYAs) with acute lymphoblastic leukemia (ALL). Cancer 2014 Jul 17. [Epub ahead of print]

24. Advani AS, Sanford B, Luger S, et al. Frontline treatment of acute lymphoblastic leukemia (ALL) in older adolescents and young adults (AYAs) using a pediatric regimen is feasible: toxicity results of the prospective US intergroup trial C10403. Presented at 55th ASH Annual Meeting, New Orleans, December 2013.

25. Hough R, Rowntree C, Wade R, et al. Impact of age on toxicity associated with chemotherapy for acute lymphoblastic leukemia (ALL): results from the UK prospective study UKALL2003. Presented 
at 55th ASH Annual Meeting, New Orleans, December 2013.

26. Plourde PV, Jeha S, Hijiya N, et al. Safety profile of asparaginase Erwinia chrysanthemi in a large compassionate-use trial. Pediatr Blood Cancer 2014;61:1232-8.

27. Breitenbach K, Stock W. Intergroup Trial C10403: a pediatric treatment approach to improve outcomes in adolescents and young adults with acute lymphoblastic leukemia. JAYAO 2011;1:107-8.

28. Stock W. Disparity in outcome of young adults with ALL (response). Blood 2009;113:1862.

29. Earl M. Incidence and management of asparaginase-associated adverse events in patients with acute lymphoblastic leukemia. Clin Adv Hematol Oncol 2009;7:600-6.

30. Hunault-Berger M, Chevallier P, Delain M, et al. GOELAMS (Groupe Ouest-Est des Leucémies Aiguës et Maladies du Sang). Changes in antithrombin and fibrinogen levels during induction chemotherapy with L-asparaginase in adult patients with acute lymphoblastic leukemia or lymphoblastic lymphoma. Use of supportive coagulation therapy and clinical outcome: the CAPELAL study. Haematologica 2008;93:1488-94.

31. Raetz EA, Salzer WL. Tolerability and efficacy of L-asparaginase therapy in pediatric patients with acute lymphoblastic leukemia. J Pediatr Oncol 2010:32:554-63.

32. Lowas SR, Marks D, Malempati S. Prevalence of transient hyperglycemia during induction chemotherapy for pediatric acute lymphoblastic leukemia. Pediatr Blood Cancer 2009;52:814-8.

33. Sonabend RY, McKay SV, Okcu MF, et al. Hyperglycemia during induction therapy is associated with increased infectious complications in childhood acute lymphocytic leukemia. Pediatr Blood Cancer 2008;51:387-92.

34. Advani A, Earl M, Douer D, et al. Toxicities of intravenous (IV) pegasparaginase (ONCASPAR) in adults with acute lym- phoblastic leukemia (ALL). Blood 2007;110:Abstract 2811.

35. Sonabend RY, McKay SV, Okcu MF, et al. Hyperglycemia during induction therapy is associated with poorer survival in children with acute lymphocytic leukemia. J Pediatr 2009;155:73-8.

36. Raja RA, Schmiegelow K, Frandsen TL. Asparaginase-associated pancreatitis in children. Br J Haematol 2012;159:18-27.

37. Knoderer HM, Robarge J, Flockhart DA. Predicting asparaginase-associated pancreatitis. Pediatr Blood Cancer 2007;49: 634-9.

38. Bostrom B. Successful management of extreme hypertriglyceridemia from pegaspargase with omega-3. Pediatr Blood Cancer 2012;59:350.

39. Tong WH, Pieters R, van der Sluis IM. Successful management of extreme hypertriglyceridemia in a child with acute lymphoblastic leukemia by temporarily omitting dexamethasone while continuing asparaginase. Pediatr Blood Cancer 2012; 58:317-8.

40. Rizzari C, Conter V, Starý J, et al. Optimizing asparaginase therapy for acute lymphoblastic leukemia. Curr Opin Oncol 2013;25:S1-9.

41. Zalewska-Szewczyk B, Andrzejewski W, Mlynarski W, et al. The anti-asparagines antibodies correlate with L-asparagines activity and may affect clinical outcome of childhood acute lymphoblastic leukemia. Leuk Lymphoma 2007;48:931-6.

42. Zalewska-Szewczyk B, Gach A, Wyka K, et al. The cross-reactivity of anti-asparaginase antibodies against different Lasparaginase preparations. Clin Exp Med 2009;9:113-6.

43. Salzer WL, Asselin B, Supko JG, et al. Erwinia asparaginase achieves therapeutic activity after pegaspargase allergy: a report from the Children's Oncology Group. Blood 2013;122:507-14.

44. Asselin BL. The right dose for the right patient. Blood 2012;119:1617-8.

45. Kawedia JD, Liu C, Pei D, et al.
Dexamethasone exposure and asparaginase antibodies affect relapse risk in acute lymphoblastic leukemia. Blood 2012;11: 1658-64.

46. Douer D, Yampolsky H, Cohen LJ, et al. Pharmacodynamics and safety of intravenous pegaspargase during remission induction in adults aged 55 years or younger with newly diagnosed ALL. Blood 2007;109:2744-50.

47. Grace RF, Dahlberg SE, Neuberg D, et al. The frequency and management of asparaginase-related thrombosis in paediatric and adult patients with acute lymphoblastic leukaemia treated on DanaFarber Cancer Institute consortium protocols. Br J Haem 2011;152:452-9.

48. Mitchell L, Lambers M, Flege $\mathrm{S}$, et al. Validation of a predictive model for identifying an increased risk for thromboembolism in children with acute lymphoblastic leukemia: results of a multicenter cohort study. Blood 2010;115:4999-5004.

49. Putti MC, Randi ML. Thrombotic complications in children with haematologic malignancies. Thromb Res 2010;125:S151-4.

50. Santoro N, Colombini A, Silvestri D, et al. Screening for coagulopathy and identification of children with acute lymphoblastic leukemia at a higher risk of symptomatic venous thrombosis: an AIEOP experience. J Pediatr Hematol Oncol 2013;35: $348-55$.

51. Mitchell LM. Andrew K. Hanna T, et al. Trend to efficacy and safety using antithrombin concentrate in prevention of thrombosis in children receiving $\mathrm{l}$ asparaginase for acute lymphoblastic leukemia: results of the PARKAA study. Thromb Haemost 2003;90:235-44.

52. Qureshi A, Mitchell C, Richards S, et al. Asparaginase-related venous thrombosis in UKALL 2003- re-exposure to asparaginase is feasible and safe. Br J Haematol 2010;149:410-3. 